\title{
Neurosurgery Oral Board Review by Jonathan S. Citow et al. (2019) 376 pp, 335 illustrationsPaperback/softback,ISBN: 9781684201266 Thieme publishers New York/Stuttgart.
}

\author{
Vittorio Stumpo ${ }^{1} \cdot$ Victor E. Staartjes ${ }^{2}$ \\ Received: 20 February 2020 / Accepted: 21 February 2020 / Published online: 3 April 2020 \\ (C) Springer-Verlag GmbH Austria, part of Springer Nature 2020
}

Neurosurgery Oral Board Review, published by Thieme in its 3rd edition, is a 376 pages pocket-sized paperback book to prepare examinees for the American Board of Neurological Surgery (ABNS) oral exam. The oral exam is the last step after successful completion of neurosurgical residency in an ACGME-accredited program and of the written ABNS exam. While the latter examines theoretical knowledge, the oral exam consists of an interactive interview where the applicants discuss pre-, peri-, and postoperative aspects of clinical cases with the examining board.

It is immediately clear at first glance how the strength of this book is in its brevity and easy-to-read format, which favors quick consultation in between more organic readings and most importantly during the reader's busy schedule while approaching the exam.

The first half of the book is structured into 3 sections: spinal disorders, cranial disorders, and miscellaneous topics, subdivided in 5 chapters. These 15 chapters address in a schematic way by bullet points, lists, and tables the most important notions for each topic and are enriched by sparse pertinent images as needed, schematic anatomical drawings (which to some extent could be more accurate but serve the purpose of easy mnemonics), clinical cases, and "Helpful Hints" boxes at the end of each chapter. The different subjects are discussed in a balanced manner.

The second half of the book is divided into 8 categories of case vignettes (general cases, neurology mimics, spine, vascular, tumor, pediatrics, functional, and peripheral nerve), presenting 10 cases, each reminiscent of those that could be an

Victor E. Staartjes

victoregon.staartjes@usz.ch

Università Cattolica del Sacro Cuore, Rome, Italy

2 Department of Neurosurgery, Clinical Neuroscience Center, University Hospital Zurich, University of Zurich, Frauenklinikstrasse 10, 8091 Zurich, Switzerland object of evaluation in the ABNS oral exam. Notably, as each case is developed in an interactive form, and to which each part of the case presentation the expected answer is provided, pertinent suggested references are reported-allowing the reader to address further and in more the subject discussed. This organization allows the examinee to decide whether to practice on the case presented by providing his own answer (and then check it again) or to go through the entire case and then review whether his or her knowledge would have been appropriate to answer correctly during the interview. With this method, the suggested readings listed throughout the text are an additional point of strength. In addition to addressing diagnosis and management, the authors correctly focus on possible complications arising in these clinical scenarios as complications management; plus in addition to representing a common event in clinical practice, this is also the specific object of evaluation in the oral exam. The last 30 pages of the book constitute a quick reference guide made up of classification tables, management flowchart, and concise notes about results of cornerstone studies that can be rapidly skimmed as needed and also include a glossary and an index. Lastly, a complementary online version of the book is available and can be accessed after a registration.

To summarize, the authors are to be applauded for providing schematic notes for easy consultation, study planning, and quick review in exam preparation, elegantly organized, covering all topics of the examination spanning from cranial and spinal pathology, and also including topics such as functional and pain neurosurgery, critical care, and neuroanesthesia. Consultation of more detailed textbooks and sources while preparing for the exam, which is suggested and encouraged throughout the book, remains paramount for a proper preparation. This book developed for ABNS oral exam preparation can also constitute a useful support resource for young neurosurgeons in countries other than the USA. 